\title{
Teacher reports of hypoactivity symptoms reflect slow cognitive processing speed in primary school children
}

\author{
Astri J. Lundervold • Maj-Britt Posserud • \\ Anne-Karin Ullebø $\cdot$ Lin Sørensen · \\ Christopher Gillberg
}

Received: 29 August 2009/Accepted: 15 December 2010/Published online: 29 December 2010

(C) The Author(s) 2010. This article is published with open access at Springerlink.com

\begin{abstract}
The mediating effect of cognitive processing speed on the ability of a primary school child to achieve his/her full potential of intellectual functioning emphasizes the importance of methods to detect "slow" children. Primary school teachers may be the first to have concerns about inattentive pupils who show symptoms of hypoactivity, but may find the symptoms difficult to interpret. In the present study we ask if a primary school teacher's report of hypoactivity symptoms can be explained by the child's performance on tests of processing speed. The 255 children included in the present study were part of the first wave of the Bergen Child Study, in which teachers completed a questionnaire including two hypoactivity items from the Five to Fifteen (FTF) questionnaire. Processing speed was measured by the Processing Speed Index (PSI) from the WISC-III, 1-2 years after the teacher rating. Teachers reported "certainly true" on at least one FTF item of hypoactivity for $11.8 \%$ of the children. These children obtained lower scores on the PSI than the remaining children in the sample. The PSI accounted for a considerable proportion of the variance of teacher reports on the FTF
\end{abstract}

\author{
A. J. Lundervold $(\bowtie) \cdot$ L. Sørensen \\ Department of Biological and Medical Psychology, \\ University of Bergen, Bergen, Norway \\ e-mail: astri.lundervold@psych.uib.no \\ C. Gillberg \\ Gillberg Centre, Sahlgrenska Academy, Gothenburg, Sweden
}

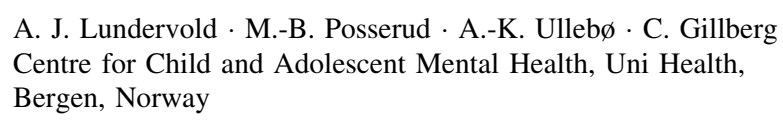

M.-B. Posserud · L. Sørensen

Department of Child and Adolescent Psychiatry,

Haukeland University Hospital, Bergen, Norway item "difficulty getting started on a task/activity". The risk of a PSI score below 85 was increased in children with teacher-reported hypoactivity symptoms. The results indicate that teacher reports of hypoactivity symptoms reflect slow cognitive processing speed and should be followed up by a psychometric examination. Still, future studies are needed to improve detection and treatment of children with slow processing speed.

Keywords Hypoactivity · Processing speed · Teacher-reports $\cdot$ Children

\section{Introduction}

Many school tasks necessitate quick decisions, and children with a slow cognitive processing speed may be disadvantaged if they have to solve tasks at the same tempo as their peers. The importance of such slowness to the academic development was emphasized in a study by Fry and Hale [1], showing that cognitive processing speed is a mediator of the age-related increase in intellectual function. This was supported in a more recent study, showing that performance on tests of cognitive processing speed explains a significant part of performance on a standardized test of intellectual functioning [2]. Furthermore, poor results on such tests have been related to both behavioural [3-5] and emotional disorders [6-8]. These findings emphasize the importance of clinical methods for early detection of slow cognitive processing speed in children.

A primary school teacher may be the first to notice what may reflect slowness due to impaired cognitive processing speed. She/he will probably be worried if a child is slow to initiate a task or seems to lack the energy to fully take part in academic activities, characteristics that have been 
referred to as symptoms of hypoactivity [9]. However, she/ he may find it difficult to interpret the aetiology and significance of such symptoms and to meet the needs of the child. If the child is not referred to a more formal cognitive assessment, including psychometric tests of processing speed, the question will remain whether the child's problems should be defined as a cognitive problem.

This motivated the present study to investigate the association between primary school teacher's report of symptoms of hypoactivity and the child's cognitive processing speed as measured by psychometric tests. The participants were all part of the first wave of the Bergen Child Study (BCS), a population-based study of mental health and development of children 7-9 years at inclusion. Teachers of all children were asked to complete a questionnaire including two items from the Five to Fifteen (FTF) questionnaire [9] ("appears slow, sluggish and lacking energy" and "difficulty getting started on a task/ activity") which are part of the hypoactivity symptom domain of FTF. A subsample from the BCS population participated in a clinical study including a psychometric measure of cognitive processing speed. We hypothesised that children with high scores on teacher-reported FTF symptoms of hypoactivity in this subsample would also show slow performance on the processing speed measure and that processing speed performance would contribute substantially to explain the variance of the teacher reports on the FTF items. Finally, we investigated the risk of being defined with hypoactivity symptoms by the teacher if the PSI score was below the normal range (i.e., <85).

\section{Methods}

\section{The Bergen Child Study}

The present research is part of the first wave of BCS, a longitudinal population-based study of children $(N=9,430)$ attending second-fourth grade (7-9 years of age) in all schools in Bergen, Norway, at inclusion (October 2002). The study was approved by the Regional Committee for Medical Research Ethics in Western Norway and by the Privacy Ombudsman for Research, Norwegian Social Science Data Services Ltd. The protocol, population, and attrition rates in the first wave of BCS have been described in detail in separate publications [cfr. 10-12].

Briefly, the first wave of the BCS included three stages. In stage 1, a questionnaire screening for behaviour problems and psychiatric disorders was sent to all parents and teachers of the whole Bergen population $(n=9,400)$. The questionnaire included-among other instruments-the two items from the FTF hypoactivity symptom domain [9]. The BCS also included the Strengths and Difficulties score (SDQ), the DSM-IV symptoms of ADHD as part of a modified version of the Swanson, Nolan and Pellham (SNAP-IV) questionnaire [13]; the Autism Spectrum Screening Questionnaire [10, 14], questions addressing obsessive-compulsive symptoms [15] and questions on tics symptoms [16]. In stage 2, parents of children defined as screen positive (defined in Fig. 1) in stage 1 and a sample of screen negative children were interviewed according to the Development And Well-Being Assessment (DAWBA, http://www.Dawba.com) [17]. The DAWBA is a fully structured interview with open questions performed by trained lay interviewers and scored by experienced clinicians [11]. A stage 3 included an in-depth psychiatric and neuropsychological assessment of a subsample of "DAWBA positive" (i.e. children with a diagnosis) and "DAWBA negative" children from stage 2.

The subsample in focus in the present study consisted of all children participating in the third stage, altogether 304 children from the Bergen population, 8-11 years of age at the time of examination. They were invited together with their parents to participate in an examination including the Wechsler Intelligence Scale for Children, third edition (WISC-III, 18), as well as supplementary tests of attention, memory and motor function and a clinical diagnostic interview [19]. Ten of the children were excluded from the present study either because they could not perform the WISC-III test $(N=1)$ or because their teachers had not checked both FTF hypoactivity items in the first stage of the BCS $(N=9)$. All children with a tested full-scale IQ below 70 were excluded from participation in the present study $(N=39)$, leaving a final sample of 255 children (94 girls, 161 boys) for the present study. The number of children participating in each of the three stages is illustrated in Fig. 1. Of the 234 included children with information from the DAWBA interview, 68 children (22 girls) obtained a DSM-IV diagnosis.

\section{The hypoactivity items in the BCS questionnaire}

The two hypoactivity items, "appears slow, sluggish or lacking energy" and "difficulty getting started on a task/ activity" from the FTF questionnaire were only included in the teacher version. They were scored as 0 ("not true"), 1 ("somewhat true") or 2 ("certainly true"). Although the two items have been shown to have good internal consistency, with a Cronbachs alpha of 0.78 [9], the two items assess different aspects of hypoactivity that may lead to impairment for different reasons. For instance, difficulty initiating a task may be due to a reluctance to start, but it could equally well be caused by distractibility/lack of focused attention, or a more general cognitive impairment. 


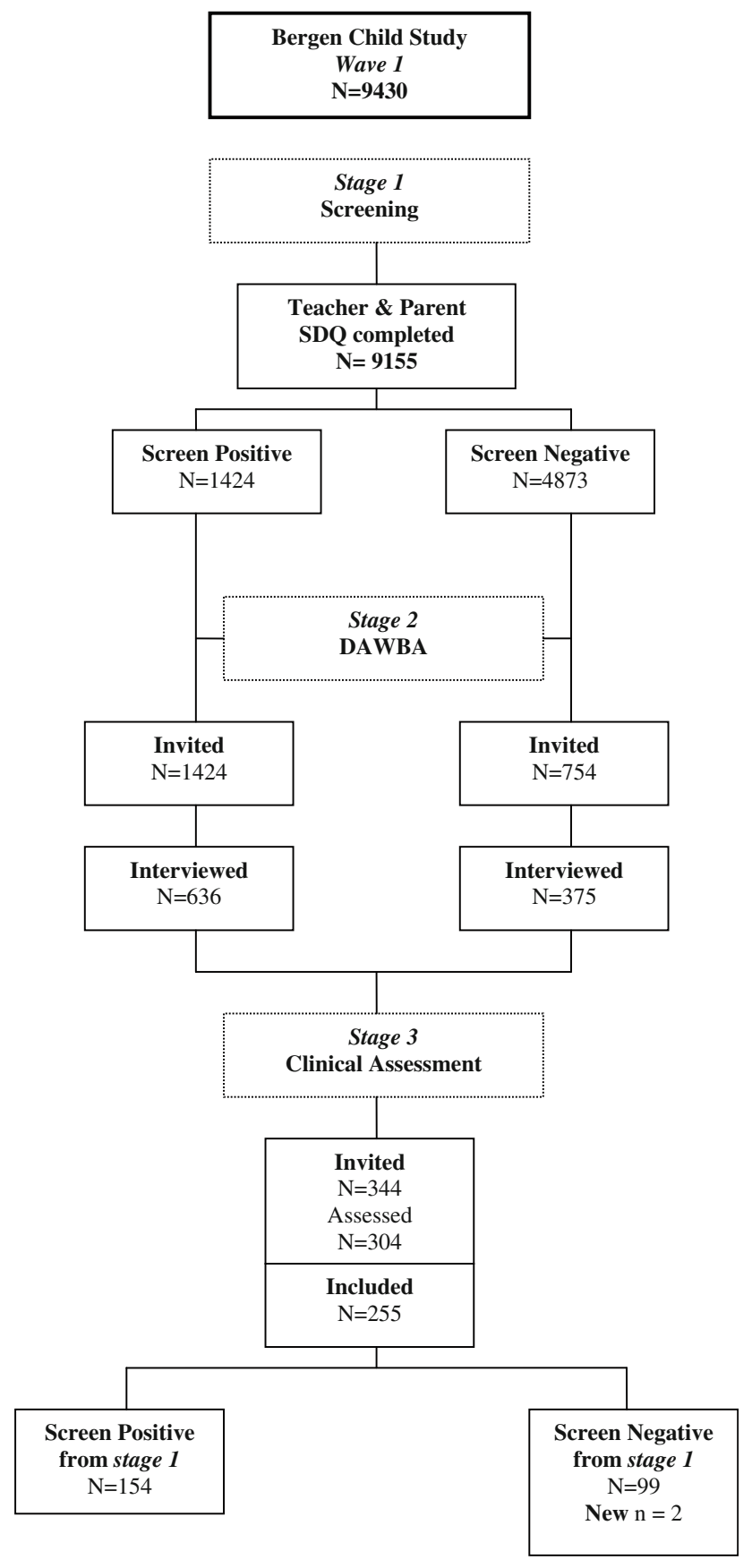

Fig. 1 Flowchart describing the selection of participants in the present study. Screen positive 1 the SDQ total difficulty score exceeded the 90th percentile cut off according to parents of teachers, 2 there was a severe impairment according to parents or teachers on the SDQ impact section, or 3 the score on one of the other scales included in the questionnaire exceeded the 98th percentile

Some teachers may report one but not both symptoms. Therefore, the two items were included separately in some analyses. In the main analyses, a child was defined as impaired if the teacher reported "certainly true" on at least one of the two items.
Cognitive processing speed

In the WISC-III [18], the subtests "Symbol Search" and "Digit Symbol" are included in a Processing Speed Index (PSI). The PSI was used as the measure of processing speed in the present study. The other WISC-III indices, verbal comprehension (VCI), perceptual organization (POI), and freedom from distractibility (FFDI) were also included with a view to investigate the specificity of the decline of the PSI in children with a "certainly true" teacher report on at least one of the two FTF items. The WISC-III was administered and scored by trained technicians in a quiet room designed for testing. Scaled scores on the four indices and the Full Scale IQ (FSIQ) scores were derived from Swedish norms [20].

\section{Statistics}

For statistical analyses we used the SPSS package, version PASW 17.2. Descriptive statistics were used to present the frequency of teacher reports of hypoactivity symptoms. The children with a "certainly true" report on at least one of the two FTF items were compared with their peers on the WISC-III indices by using an Independent Samples $t$ test. Effect sizes ( $d$ values) were calculated using pooled standard deviation. A general guideline for interpreting the $d$ value is that a $d$ of 0.20 is small, a $d$ of 0.50 is moderate and a $d$ of 0.80 is large [21].

A logistic regression analysis was run to investigate whether teacher ratings of the two FTF hypoactive symptoms could be explained by the child's result on the PSI. Three different analyses were run, including children defined as impaired (i.e., children with a "certainly true" score on at least one of the two FTF items) as a dependent variable in the first analysis, and then two separate analyses of children with a "certainly true" report on the items "appears slow, sluggish or lacking energy" and "difficulty getting started on a task/activity", respectively.

To investigate the risk of being defined as hypoactive if the PSI score was below normal range, the PSI score was categorized into a level below 85 and a level equal to or above 85 .

\section{Results}

Teacher reports on the FTF items

In the subsample of 255 children, the correlation between the two FTF items was statistically significant $(r=0.384$, $p<0.001$ ). The responses "somewhat true" or "certainly true" had a lower frequency on the FTF item "appears to be slow, sluggish or lacking energy" (9.8 and 2.7\%, 
Table 1 Mean (SD) values for the four WISC-III factors for children with a "certainly true" report on either or one of the two FTF items

\begin{tabular}{lllll}
\hline & VCI & POI & FFDI & PSI \\
\hline Impaired $(n=30)$ & $91.0(12.5)$ & $95.8(12.2)$ & $91.6(13.2)$ & $84.5(14.3)$ \\
Non-impaired $(n=255)$ & $93.6(12.9)$ & $98.2(13.8)$ & $96.5(16.5)$ & $95.4(16.9)^{*}$ \\
Sluggish $(n=7)$ & $89.4(18.3)$ & $90.9(14.4)$ & $89.9(16.1)$ & $85.0(14.1)$ \\
Not sluggish $(n=248)$ & $93.4(12.7)$ & $98.1(13.6)$ & $96.1(16.2)$ & $94.4(17.0)$ \\
Slow to initiate $(n=26)$ & $91.0(11.5)$ & $95.7(12.0)$ & $90.3(13.5)$ & $82.9(14.5)$ \\
Not slow to initiate $(n=229)$ & $93.5(13.0)$ & $98.1(13.8)$ & $96.5(16.4)$ & $95.4(16.7)^{* *}$ \\
\hline
\end{tabular}

Impaired, "certainly true" report on at least one of the two FTF items; Sluggish, "certainly true" report on the item "appears to be slow, sluggish or lacking energy"; Slow to initiate, "certainly true" report on the item "difficulty getting started on a task/activity"

$V C I$ verbal comprehension index, $P O I$ perceptual organisation index, FFDI freedom from distraction index, PSI processing speed index

$* p=0.01, * * p<0.001$

respectively) than on the FTF item "difficulty getting started on a task/activity" (25.1 and $10.2 \%$, respectively). Thirty of the children were reported with a "certainly true" response on at least one of the FTF items (11.8\%). Such report was significantly more frequent among boys $(n=27)$ than girls $\left(X^{2}=10.54, d f=1, p=0.001\right)$. The highest number of children was found in the group reported with a "somewhat true" answer on the FTF item "difficulty getting started on a task/activity". Only three children, all boys, obtained a definite score on both FTF items.

\section{Cognitive processing speed}

Table 1 shows the results on the index scores from the WISC-III test. The sample of children reported with a "certainly true" score on at least one of the FTF items $(n=30)$ obtained much lower results on the PSI (mean $=84.5, \mathrm{SD}=14.3)$ than the other children in the sample $($ mean $=95.4, \mathrm{SD}=16.8, t=3.39, d f=253$, $p=0.001, d=0.66$ ). None of the other indices significantly differentiated those with a "certainly true" report on either of the two FTF items from the other children. The item "difficulty getting started on a task/activity" accounted for the significant difference in terms of poor PSI results (cfr. Table 1).

\section{Prediction of reported hypoactivity symptoms}

A logistic regression analysis with children obtaining a "certainly true" score on at least one of the two FTF items as the dependent variable (marked as "Impaired" in Table 2) showed a statistically significant contribution of processing speed. Including the FTF item "appears to be slow, sluggish or lacking energy" as the dependent variable resulted in non-significant results for processing speed. The contribution of processing speed was statistically significant when the item "difficulty getting started on a task/ activity" was included as the dependent variable.
Table 2 Contribution of processing speed to explain if a child was reported with a "certainly true" score on the two FTF items

\begin{tabular}{|c|c|c|c|c|c|c|}
\hline \multirow[b]{2}{*}{ Predictor } & \multicolumn{2}{|l|}{ Impaired } & \multicolumn{2}{|c|}{ Sluggish } & \multicolumn{2}{|c|}{ Slow to initiate } \\
\hline & B & SE & B & SE & B & SE \\
\hline Processing speed & $-0.039^{*}$ & 0.013 & -0.31 & 0.021 & $-0.045^{*}$ & 0.014 \\
\hline
\end{tabular}

Impaired, "certainly true" report on at least one of the two FTF items; Sluggish, "certainly true" report on the item "appears to be slow, sluggish or lacking energy"; Slow to initiate, "certainly true" report on the item "difficulty getting started on a task/activity"

$B$ beta value, $S E$ standard error of beta

$* p<0.01$

\section{Children with a PSI score below 85}

A higher proportion of children with a PSI score below 85 obtained a "certainly true" report from their teachers on at least one of the FTF items (46.7\%) than their peers [27.1\%, $\mathrm{OR}=1.72(\mathrm{CI}=1.11-2.67)]$. The risk was increased both when teachers reported "certainly true" on the FTF item "appears to be slow, sluggish or lacking energy" $[\mathrm{OR}=1.48 \quad(\mathrm{CI}=0.614-3.55)]$ and on the FTF item "difficulty getting started on a task/activity" $[\mathrm{OR}=2.02$ $(\mathrm{CI}=1.33-3.06)]$.

\section{Discussion}

An important finding in the present study was that a wellrecognized measure of processing speed differentiated children recognized by teachers as having symptoms of hypoactivity from their peers and that cognitive processing speed contributed substantially to the variance in teacher reports of hypoactivity symptoms. Actually, the children reported with hypoactivity symptoms had a close to twofold higher risk to obtain a score below 85 than their peers. This held even though the teacher ratings of hypoactivity 
symptoms had been performed more than 1 year prior to the psychometric testing. Somewhat surprisingly, the teacher reports on the FTF item "appears to be slow, sluggish or lacking energy" were not explained by the PSI measure from WISC-III. This was only true for the FTF item "difficulty getting started on a task/activity", however, the conclusion should be modified by the fact that very few children $(n=7)$ were rated with a "certainly true" report on the "appears to be slow, sluggish or lacking energy" item.

The unique contribution of the present study was to show that teacher reports of hypoactivity symptoms may reflect an early sign of the child's cognitive processing tempo, a cognitive function that has been shown to overlap with cognitive control functions [22], needed in solving tasks containing a conflict between salient stimulus [23] and necessary for using the working memory, set-shifting, and response inhibition abilities [24]. The importance of this finding is emphasized by the fact that early recognition of slow processing speed in a child can help to adjust the teaching in lowering the demand of tempo in information processing and thereby help the child to solve complex tasks that require the use of cognitive control abilities. A child not meeting the tempo demands in information processing might easily get stressed and develop symptoms of anxiety for not being able to meet the requirements at school. Such anxiety will probably in itself lower the cognitive ability to make quick decisions in a task, further increase problems with processing speed [8] and decrease the ability to solve complex tasks because of a stress response in the brain limiting the neural resources for cognitive processing [25].

However, to obtain a firm conclusion about the association between processing speed and hypoactivity symptoms in children with symptoms within and across diagnostic categories, it is necessary to include a larger number of children than in the present study. One should also consider including a wider range of tasks assessing different aspects of the processing speed ability. The question remains as to whether the PSI measure from WISC-III measures the same underlying construct as do other tasks of cognitive speed, and if healthy children and children within different diagnostic categories are affected on different aspects of PS. Another limitation in the present study is the inclusion of only two items to measure hypoactivity symptoms. The two items seemed to tap different types of problem and are therefore not appropriate to identify a separate hypoactivity factor. However, by using these two items we were able to show that the teacher responses were significantly explained by the children's cognitive processing speed, meaning that teachers should take more note of these types of child characteristics and refer for more formal testing of cognitive processing speed.

Finally, the design of the present study did not allow for any conclusion about the impact of teacher reports of hypoactivity symptoms and slow processing speed on the child's future mental health. In the age group included in the present study, slow processing speed would be expected to be associated with academic failure, which might then result in low self-esteem and emotional problems. In a parallel manner, a primary school child with an externalizing disorder and slow processing speed due to problems initiating an activity may receive a lot of negative feedback from teachers due to their misinterpretation of the problem (e.g. "he is unwilling to work"). This may then result in emotional problems, putting the child at risk of developing both types of disorders. Recent studies have shown promising results by introducing training programs when the child enters school [26], programs that may help children with such cognitive problems to obtain a more successful mental health development. This emphasizes the importance of increased awareness from primary school teachers to notice what may reflect slowness due to impaired cognitive processing speed.

\section{Conclusion}

The main contribution of the present study was to show that teacher reports of hypoactivity symptoms detected children with slow cognitive processing speed on psychometric test measures, suggesting that such reports should be followed up by formal assessment of the child's cognitive function. However, reports on the two FTF items were not able to identify all children with slow processing speed. The takehome message of the present study is the importance of slow processing speed - and its implications-in the daily life of children, and that primary school teachers need to be well trained in order to identify and help children observed to be slow to initiate a task or who seem to lack the energy to fully take part in activities at school.

Acknowledgments The present study was funded by the Norwegian Directorate for Health and Social Affairs, the Norwegian Research Council and the Western Norway Regional Health Authority. We are grateful to the children, parents and teachers participating in the BCS, and to the other members of the project group for making the study possible.

Open Access This article is distributed under the terms of the Creative Commons Attribution Noncommercial License which permits any noncommercial use, distribution, and reproduction in any medium, provided the original author(s) and source are credited.

\section{References}

1. Fry A, Hale S (2000) Relationships among processing speed, working memory, and fluid intelligence in children. Biol Psychol 54:1-34 
2. Tillman C, Bohlin G, Sørensen L, Lundervold AJ (2009) Intelligence and specific cognitive abilities in children. Arch Clin Neuropsychol 24(8):769-782

3. Weiler M, Bernstein J, Bellinger DC, Waber DP (2000) Processing speed in children with attention deficit/hyperactivity disorder, inattentive type. Child Neuropsychol 6:218-234

4. Chhabildas N, Pennington BF, Willcutt E et al (2001) A comparison of the neuropsychological profiles of the DSM-IV subtypes of ADHD. J Abnorm Child Psychol 29:529-540

5. Rucklidge J, Tannock R (2002) Neuropsychological profiles of adolescents with ADHD: effects of reading difficulties and gender. J Child Psychol Psychiatry 43:988-1003

6. Tsourtos G, Thompson JC, Stough C (2002) Evidence of an early information processing speed deficit in unipolar major depression. Psychol Med 32:259-265

7. Mezulis AH, Hyde JS, Abramson LY (2006) The developmental origins of cognitive vulnerability to depression: temperament, parenting, and negative life events in childhood as contributors to negative cognitive style. Dev Psychol 42:1012-1025

8. Schatz D, Rostain A (2006) ADHD with comorbid anxiety: a review of the current literature. J Atten Disord 10(2):141-149

9. Kadesjö B, Janols L-O, Korkman M et al (2004) The FTF (Five to Fifteen): the development of a parent questionnaire for the assessment of ADHD and comorbid conditions. Eur Child Adolesc Psychiatry 13(3):3-13

10. Posserud M, Lundervold AJ, Gillberg C (2006) Autistic features in a total population of 7-9-year-old children assessed by the ASSQ (Autism Spectrum Screening Questionnaire). J Child Psychol Psychiatry 47(2):167-175

11. Heiervang E, Stormark KM, Lundervold AJ et al (2007) The Bergen Child Study of mental health in school-age children. Psychiatric disorders according to the Development and WellBeing Assessment: prevalence, risk factors and service use. J Am Acad Child Adolesc Psychiatry 46(4):438-447

12. Stormark K, Heiervang E, Heimann M, Lundervold AJ, Gillberg C (2008) Predicting nonresponse bias from teacher ratings of mental health problems in primary school children. J Abnorm Child Psychol 36(3):411-419

13. Swanson J, Kraemer H et al (2001) Clinical relevance of the primary findings of the MTA: success rates based on severity of
ADHD and ODD symptoms at the end of treatment. J Am Acad Child Adolesc Psychiatry 40(2):168-179

14. Ehlers S, Gillberg C (1993) The epidemiology of Asperger syndrome. A total population study. J Child Psychol Psychiatry 34(8):1327-1350

15. Thomsen PH (1998) Obsessive-compulsive disorder in children and adolescents. Clinical guidelines. Eur Child Adolesc Psychiatry $7(1): 1-11$

16. Apter A, Pauls DL, Bleich A et al (1993) An epidemiologic study of Gilles de la Tourette's syndrome in Israel. Arch Gen Psychiatry 50(9):734-738

17. Goodman R, Ford T, Richards H, Gatward R, Meltzer H (2000) The Development And Well-Being Assessment: description and initial validation of an integrated assessment of child and adolescent psychopathology. J Child Psychol Psychiatry 41:645-655

18. Wechsler D (1991) Wechsler Intelligence Scale for children, 3rd edn. Psychological Corporation, San Antonio

19. Kaufman J, Birmaher B, Brent D et al (1997) Schedule for affective disorders and schizophrenia for school-age childrenpresent and Lifetime version (K-SADSPL): initial reliability and validity data. J Am Acad Child Adolesc Psychiatry 36(7):980-988

20. Sonnander S, Ramund B, Smedler AC (1998). WISC-III manual, Svenske Psykologforlaget

21. Cohen J (1988) Statistical power and analysis for the behavioral sciences. Lawrence Erlbaum Associates, Hillsdale

22. Miyake A, Friedman NP, Emerson MJ, Witzki AH, Howerter A, Wager TD (2000) The unity and diversity of executive functions and their contributions to complex "Frontal Lobe" tasks: a latent variable analysis. Cogn Psychol 41(1):49-100

23. Miller EK, Cohen J (2001) An integrative theory of prefrontal cortex function. Annu Rev Neurosci 24:167-202

24. Corbetta M, Shulman GL (2002) Control of goal-directed and stimulus-driven attention in the brain. Nat Rev Neurosci 3:201-215

25. Eysenck M, Derakshan N, Santoset R, Calvo MG (2007) Anxiety and cognitive performance: attentional control theory. Emotion 7(2):336-353

26. McNab F, Varrone A, Farde L, et al. (2009) Changes in cortical dopamine D1 receptor binding associated with cognitive training. Science 323(5915): 800-802 